
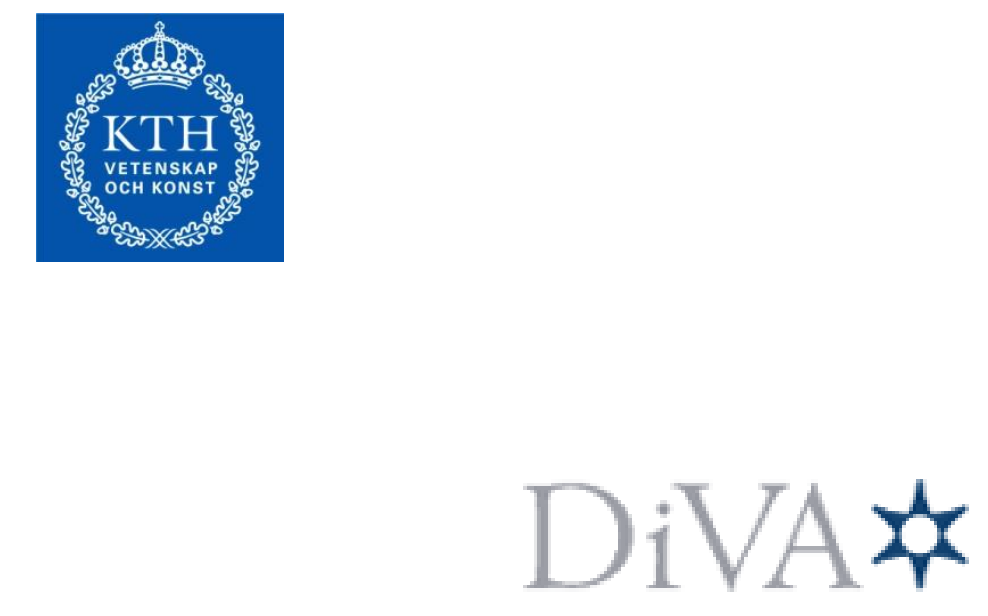

http://kth.diva-portal.org

This is an author produced version of a paper published in Statistical Signal Processing Workshop (SSP), 2012 IEEE.

This paper has been peer-reviewed but does not include the final publisher proofcorrections or proceedings pagination.

(C) 2012 IEEE. Personal use of this material is permitted. Permission from IEEE must be obtained for all other uses, in any current or future media, including reprinting/republishing this material for advertising or promotional purposes, creating new collective works, for resale or redistribution to servers or lists, or reuse of any copyrighted component of this work in other works.

Citation for the published paper:

Gudmundson, E., Wirfält, P. Jakobsson, A., Jansson, M. An esprit-based parameter estimator for spectroscopic data. Statistical Signal Processing Workshop (SSP), 2012 IEEE Access to the published version may require subscription. Published with permission from: IEEE 


\title{
AN ESPRIT-BASED PARAMETER ESTIMATOR FOR SPECTROSCOPIC DATA
}

\author{
Erik Gudmundson*†, Petter Wirfält*, Andreas Jakobsson ${ }^{\dagger}$, and Magnus Jansson* \\ *ACCESS Linnaeus Center, Signal Processing, KTH Royal Institute of Technology, Sweden \\ ${ }^{\dagger}$ Dept. of Mathematical Statistics, Lund University, Lund, Sweden
}

\begin{abstract}
The pulse spin-locking sequence is a common excitation sequence for magnetic resonance and nuclear quadrupole resonance signals, with the resulting measurement data being well modeled as a train of exponentially damped sinusoidals. In this paper, we derive an ESPRIT-based estimator for such signals, together with the corresponding Cramér-Rao lower bound. The proposed estimator is computationally efficient and only requires prior knowledge of the number of spectral lines, which is in general available in the considered applications. Numerical simulations indicate that the proposed method is close to statistically efficient, and that it offers an attractive approach for initialization of existing statistically efficient gradient or search based techniques.
\end{abstract}

Index Terms - Parameter estimation; damped sinusoids; subspace techniques; multidimensional signal processing; NQR; NMR

\section{INTRODUCTION AND DATA MODEL}

Spectral estimation is a classical problem which has found application in a wide variety of fields, such as astronomy, medical imaging, radar, and spectroscopic techniques (for example nuclear magnetic resonance, NMR, and nuclear quadrupole resonance, NQR). Subspaced-based estimators form an important class of spectral estimation methods that have proven to be useful for estimation of both damped and undamped sinusoidal signals (see, e.g., [1, 2]). Even though much work has been done on the estimation of damped and undamped sinusoids, there are only a few algorithms dealing with structured data models able to fit data produced by magnetic and quadrupolar resonance techniques. Such measurements are often resulting from the use of pulse spin-locking (PSL) sequences, which will then induce a fine structure into the signals. The PSL sequence consists of a preparatory pulse and a train of refocusing pulses, where the time between two consecutive refocusing pulses is $2 \tau$, as illustrated in Fig. 1. As discussed in [3, 4], the signal resulting from a PSL excitation can be well modeled as

$$
y_{m, t}=x_{m, t}+w_{m, t},
$$

where $m=0, \ldots, M-1$ denotes the echo number, and $t=$ $t_{0}, \ldots, t_{N-1}$ the local time within each echo, with $t=0$ denoting the center of the current pulse, and where we assume uniform sampling intervals within each echo. Moreover, $w_{m, t}$ is an additive circular symmetric white Gaussian i.i.d. noise with variance $\sigma^{2}$, and

$$
x_{m, t}=\sum_{k=1}^{K} \alpha_{k} \exp \left(i \omega_{k} t-\beta_{k}|t-\tau|-(t+2 \tau m) \eta_{k}\right),
$$

This work was supported in part by the Swedish Research Council, Carl Trygger's foundation, and the European Research Council (ERC, grant agreement numbers 228044 and 261670). where $\alpha_{k}, \omega_{k}=2 \pi f_{k}, \beta_{k}$, and $\eta_{k}$ denote the (complex) amplitude, frequency, damping coefficient (with respect to the current transmitted pulse), and the compound, or echo train, damping coefficient for the $k$ th spectral line, respectively; additionally, $\tau$ is a design parameter (due to operator choice of pulse repetition interval) and is thus known. Common approaches for estimating the parameters for this form of data is to sum the echoes, thereby destroying the finer details resulting from the echo train decay (and causing a bias in the estimates), and then use classical approaches such as the periodogram or the matrix pencil $[1,2]$. An alternative approach was taken in [3], where a least-squares (LS) based algorithm for estimating all the unknown parameters in (2) was proposed. Such an estimate is formed using a gradient or grid-based search, but then requires a careful initialization of the various parameters, an often non-trivial task. As the search can often not be well initialized, this also implies that the resulting algorithm can be computationally demanding, especially when the data consists of several spectral lines. In this paper, we propose a computationally efficient ESPRIT-based algorithm, termed the echo train ESPRIT (ET-ESP), which requires no prior knowledge of typical parameter values, needing only knowledge of the number of present spectral lines, $K$, a number that is generally known in these applications. Furthermore, we introduce the corresponding Cramér-Rao lower bound (CRB) for the problem at hand and examine the performance of the proposed algorithm using numerical simulations.

Some words on the notation: hereafter, we denote by $\operatorname{Re}\{x\}$, $\mathbf{X}^{\mathrm{T}}, \mathbf{X}^{H}, \operatorname{diag}\{\mathbf{x}\}$, and $[\mathbf{X}]_{l, k}$, the real part of $x$, the transpose of $\mathbf{X}$, the conjugate, or Hermitian, transpose of $\mathbf{X}$, the diagonal matrix with the vector $\mathbf{x}$ along the diagonal, and the $l, k$-th element of $\mathbf{X}$, respectively.

\section{THE ECHO TRAIN ESPRIT ALGORITHM}

Let (2) be expressed as

$$
x_{m, t}=\sum_{k=1}^{K} c_{m, k} z_{k}^{t}
$$

with

$$
\begin{aligned}
& c_{m, k} \triangleq\left\{\begin{array}{ll}
\alpha_{k} \exp \left(-\beta_{k} \tau\right) \cdot \exp \left(-2 \eta_{k} \tau m\right) & \text { for } t<\tau \\
\alpha_{k} \exp \left(\beta_{k} \tau\right) \cdot \exp \left(-2 \eta_{k} \tau m\right) & \text { for } t \geq \tau
\end{array},\right. \\
& z_{k} \triangleq\left\{\begin{array}{ll}
\exp \left(i \omega_{k}\right) \cdot \exp \left(\beta_{k}-\eta_{k}\right) & \text { for } t<\tau \\
\exp \left(i \omega_{k}\right) \cdot \exp \left(-\beta_{k}-\eta_{k}\right) & \text { for } t \geq \tau
\end{array} ;\right.
\end{aligned}
$$

where it should be noted that $z_{k}$ is not a function of $m$, as all echoes share the same poles. Reminiscent of [5], the noise-free data for each 


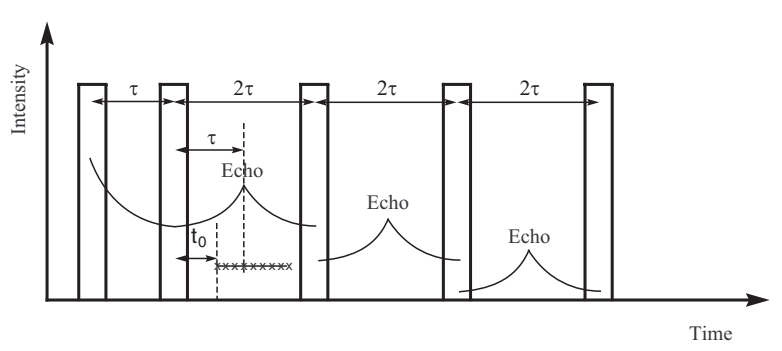

Fig. 1. The PSL sequence.

echo $m$ is then stacked into the (Hankel) matrix

$$
\mathbf{X}_{m}=\left[\begin{array}{cccc}
x_{m, t_{0}} & x_{m, t_{1}} & \cdots & x_{m, t_{L^{\prime}-1}} \\
x_{m, t_{1}} & x_{m, t_{2}} & \cdots & x_{m, t_{L^{\prime}}} \\
\vdots & \vdots & & \vdots \\
x_{m, t_{L-1}} & \cdots & \cdots & x_{m, t_{N-1}}
\end{array}\right] \in \mathbb{C}^{L \times L^{\prime}}
$$

where $L^{\prime}=N-L+1$. This (noise-free) echo data matrix may then be collected, and partitioned, as

$$
\begin{aligned}
\mathbf{X} & =\left[\begin{array}{lll}
\mathbf{X}_{0} & \cdots & \mathbf{X}_{M-1}
\end{array}\right] \\
& =\mathbf{S}\left[\begin{array}{lll}
\mathbf{C}_{0} \mathbf{T}^{\mathrm{T}} & \cdots & \mathbf{C}_{M-1} \mathbf{T}^{\mathrm{T}}
\end{array}\right]
\end{aligned}
$$

where $\mathbf{S} \in \mathbb{C}^{L \times K},[\mathbf{S}]_{l, k}=z_{k}^{t_{l-1}}, \mathbf{T} \in \mathbb{C}^{L^{\prime} \times K},[\mathbf{T}]_{l^{\prime}, k}=z_{k}^{t_{l^{\prime}-1}}$, $\mathbf{C}_{m}=\operatorname{diag}\left\{\left[\begin{array}{lll}c_{m, 1} & \ldots & c_{m, k}\end{array}\right]\right\}$, and where $c_{m, k}$ is given by (4). Thus, $\mathbf{S}$ and $\mathbf{T}$ may be factored from each $\mathbf{X}_{m}$ as in (7) due to $z_{k}$ in (5) being independent of $m$. Forming the singular value decomposition (SVD) of $\mathbf{X}$, i.e.,

$$
\mathbf{X}=\mathbf{U} \boldsymbol{\Sigma} \mathbf{V}^{H},
$$

it may be noted by comparing (7) and (8) that $\mathbf{S}$ and $\mathbf{U}$ will span the same subspace. Regrettably, only $y_{m, t}$, i.e., the noise-corrupted measurements of (2) are available, instead necessitating the forming of $\mathbf{Y}_{m}$ and $\mathbf{Y}$ from $y_{m, t}$ similarly to (6) and (7).

Typically, magnetic resonance measurements may be obtained as either one or two-sided signals. For scenarios when measurements of both the expanding and the decaying part of the signal are available, so-called two-sided echoes, as is illustrated in Fig. 1, one may partition each echo into two parts, based on (4) and (5), such that one part is formed from $t<\tau$ and the other from $t \geq \tau$. Thus,

$$
\mathbf{y}_{m}=\left[\begin{array}{lll}
y_{m, t_{0}} & \cdots & y_{m, t_{N-1}}
\end{array}\right]^{\mathrm{T}} \triangleq\left[\begin{array}{l}
\mathbf{y}_{m}^{(+)} \\
\mathbf{y}_{m}^{(-)}
\end{array}\right],
$$

where the superscripts $\mathbf{y}_{m}^{(+)}$and $\mathbf{y}_{m}^{(-)}$have been introduced to denote the expanding $(t<\tau)$ and decaying $(t \geq \tau)$ parts of $\mathbf{y}_{m}$, respectively. One then forms $\mathbf{Y}^{(+)}$using only $\mathbf{y}_{m}^{(+)}$, and similarly for $\mathbf{Y}^{(-)}$. The following estimation is then performed independently for $\mathbf{Y}^{(+)}$and $\mathbf{Y}^{(-)}$; accordingly, one gets two independent estimates for each parameter, i.e., $\hat{\gamma}_{k}^{(+)}$and $\hat{\gamma}_{k}^{(-)}$. These are then combined to form the estimate $\hat{\gamma}_{k}=\frac{1}{2} \hat{\gamma}_{k}^{(+)}+\frac{1}{2} \hat{\gamma}_{k}^{(-)}$, where $\gamma_{k}$ represents $\beta_{k}$, $\eta_{k}, \omega_{k}$, as appropriate.

Alternatively, for cases when only one-sided echoes are available, i.e., when one only obtains measurements for the decaying part $\mathbf{y}_{m}^{(-)}$, for $t \geq \tau$, the analysis is analogous, although with appropriate changes dictated by (4)-(6). In order to simplify the notation, we omit the superscript ${ }^{(-)}$in the following.
Proceeding with either forms of measurements, let $\mathbf{X}^{\uparrow}$ denote the operation of removing the bottom-most row of the matrix $\mathbf{X}$, and similarly let $\mathbf{X}^{\downarrow}$ denote removal of the top-most row. Then, it is easily seen that $\mathbf{S}^{\downarrow}=\mathbf{S}^{\uparrow} \mathbf{Z}$, where

$$
\mathbf{Z}=\operatorname{diag}\left\{\left[z_{1}, \ldots, z_{K}\right]\right\},
$$

and hence $\mathbf{U}^{\downarrow}=\mathbf{U}^{\uparrow} \boldsymbol{\Omega}$, where $\boldsymbol{\Omega}$ and $\mathbf{Z}$ are related by a similarity transformation and thus have the same eigenvalues. Using the measured data, the SVD of $\mathbf{Y}$ is formed, yielding

$$
\mathbf{Y}=\hat{\mathbf{U}} \hat{\boldsymbol{\Sigma}} \hat{\mathbf{V}}^{H}+\mathbf{W}
$$

where $\hat{\boldsymbol{\Sigma}}$ denotes the matrix formed from the $K$ largest singular values and $\hat{\mathbf{U}}$ and $\hat{\mathbf{V}}$ denote the matrices formed by the corresponding singular vectors. The residual term, $\mathbf{W}$, contains the noise. The total-least squares (TLS) estimate $\hat{\Omega}$ of $\boldsymbol{\Omega}$ may then be formed from

$$
\hat{\mathbf{U}}^{\downarrow}=\hat{\mathbf{U}}^{\uparrow} \boldsymbol{\Omega},
$$

and we may obtain estimates of the $K$ poles $\left\{\hat{z}_{k}\right\}_{k=1}^{K}$ from the eigenvalues of $\hat{\boldsymbol{\Omega}}$. Using (5), we then find $\hat{\omega}_{k}=\angle \hat{z}_{k}$ and $\widehat{\beta}_{k}+\eta_{k}=$ $-\log \left|\hat{z}_{k}\right|$. With the estimated poles, one may then, for each echo $m$, write

$$
\left[\begin{array}{ccc}
\hat{z}_{1}^{t_{0}} & \cdots & \hat{z}_{K}^{t_{0}} \\
\hat{z}_{1}^{t_{1}} & \cdots & \hat{z}_{K}^{t_{1}} \\
\vdots & \vdots & \vdots \\
\hat{z}_{1}^{t_{N-1}} & \cdots & \hat{z}_{K}^{t_{N-1}}
\end{array}\right]\left[\begin{array}{c}
c_{m, 1} \\
c_{m, 2} \\
\vdots \\
c_{m, K}
\end{array}\right]=\left[\begin{array}{c}
y_{m, t_{0}} \\
y_{m, t_{1}} \\
\vdots \\
y_{m, t_{N-1}}
\end{array}\right]
$$

which forms a regular LS problem for $\left\{c_{m, k}\right\}_{k=1}^{K}$. Using (4), we simplify the notation by introducing $d_{k} \triangleq \alpha_{k} \exp \left(\beta_{k} \tau\right)$ and then, for each spectral line $k=1, \ldots, K$, one may form the following LS problem for the estimation of $\left\{\eta_{k}\right\}_{k=1}^{K}$

$$
\left[\begin{array}{c}
\log \left|\hat{c}_{0, k}\right| \\
\vdots \\
\log \left|\hat{c}_{M-1, k}\right|
\end{array}\right]=\left[\begin{array}{cc}
1 & -2 \tau \cdot 0 \\
\vdots & \vdots \\
1 & -2 \tau \cdot(M-1)
\end{array}\right]\left[\begin{array}{c}
\log \left|d_{k}\right| \\
\eta_{k}
\end{array}\right],
$$

where $\left\{\hat{c}_{m, k}\right\}_{m=0}^{M-1}$ denote the LS solution to (13). The LS solution to (14) is readily found as

$$
\hat{\eta}_{k}=\frac{3\left[(M-1) \sum_{m=0}^{M-1} \log \left|\hat{c}_{m, k}\right|-2 \sum_{m=0}^{M-1} m \log \left|\hat{c}_{m, k}\right|\right]}{\tau M(M-1)(M+1)} .
$$

Using (5) and (15), one may then also estimate $\hat{\beta}_{k}$ as

$$
\hat{\beta}_{k}=-\left(\hat{\eta}_{k}+\log \left|\hat{z}_{k}\right|\right) .
$$

Finally, given the estimates $\left\{\hat{\beta}_{k}, \hat{\omega}_{k}, \hat{\eta}_{k}\right\}_{k=1}^{K}$, an estimate of $\alpha_{k}$, $k=1, \ldots, K$, may be formed from (1) using a maximum likelihood algorithm, which in this case coincides with the LS solution. Due to space limitations, the reader is referred to, e.g., $[3,6])$ for the details.

\section{DERIVATION OF THE CRAMÉR-RAO BOUND}

We proceed to form the Cramér-Rao Bound (CRB) for the problem at hand, stacking the data from each measurement echo as

$$
\mathbf{y}=\mathbf{x}+\mathbf{w}
$$


where

$$
\begin{aligned}
\mathbf{x} & =\left[\begin{array}{lll}
\mathbf{x}_{0}^{T} & \cdots & \mathbf{x}_{M-1}^{T}
\end{array}\right]^{T} \in \mathbb{C}^{N M \times 1} \\
\mathbf{x}_{m} & =\left[\begin{array}{lll}
x_{m, t_{0}} & \cdots & x_{m, t_{N-1}}
\end{array}\right]^{T} \in \mathbb{C}^{N \times 1} \\
\mathbf{w} & =\left[\begin{array}{lll}
w_{0, t_{0}} & \cdots & w_{M-1, t_{N-1}}
\end{array}\right]^{T} \in \mathbb{C}^{N M \times 1} .
\end{aligned}
$$

In order to simplify the notation, let

$$
\xi_{k}^{m, t} \triangleq \exp \left(i \omega_{k} t-\beta_{k}|t-\tau|-(t+2 \tau m) \eta_{k}\right),
$$

so that

$$
x_{m, t}=\sum_{k=1}^{K} \alpha_{k} \xi_{k}^{m, t} .
$$

The CRB is given as $\operatorname{CRB}(\gamma)=[\operatorname{FIM}(\gamma)]^{-1}$, where $\operatorname{FIM}(\gamma)$ denotes the Fisher information matrix given the unknown (vector) parameters $\gamma$, with

$$
\begin{array}{r}
\gamma=\left[a_{1}, \cdots, a_{K}, b_{1}, \cdots, b_{K}, \omega_{1}, \cdots, \omega_{K},\right. \\
\left.\beta_{1}, \cdots, \beta_{K}, \eta_{1}, \cdots, \eta_{K}\right]^{\mathrm{T}},
\end{array}
$$

where $a_{k}=\left|\alpha_{k}\right|$ and $b_{k}=\angle \alpha_{k}$. The CRB for a particular unknown $\gamma_{i}$ is then obtained as the $(i, i)$-th element of the CRB matrix, i.e., $\operatorname{CRB}\left(\gamma_{i}\right)=[\mathrm{CRB}]_{i i}$. From the Slepian-Bang's formula [1], it is known that

$$
\operatorname{FIM}(\gamma)=\frac{2}{\sigma^{2}} \operatorname{Re}\left\{\left(\frac{\partial \mathbf{x}}{\partial \boldsymbol{\gamma}}\right)^{H}\left(\frac{\partial \mathbf{x}}{\partial \boldsymbol{\gamma}}\right)\right\}
$$

where the derivatives may be found as

$$
\begin{aligned}
\frac{\partial x_{m, t}}{\partial a_{k}} & =\exp \left(i b_{k}\right) \xi_{k}^{m, t}, & & \frac{\partial x_{m, t}}{\partial \beta_{k}}=-|t-\tau| \alpha_{k} \xi_{k}^{m, t}, \\
\frac{\partial x_{m, t}}{\partial b_{k}} & =i \alpha_{k} \xi_{k}^{m, t}, & \frac{\partial x_{m, t}}{\partial \eta_{k}} & =-(t+2 \tau m) \alpha_{k} \xi_{k}^{m, t}, \\
\frac{\partial x_{m, t}}{\partial \omega_{k}} & =i t \alpha_{k} \xi_{k}^{m, t} . & &
\end{aligned}
$$

Reminiscent of the presentation in [7], these derivatives may be expressed on matrix form as $\partial \mathbf{x}_{m} / \partial \boldsymbol{\gamma}=\mathbf{Q}_{m} \mathbf{P}$, where

$$
\begin{aligned}
& \mathbf{Q}_{m} \triangleq\left[\begin{array}{lllll}
\boldsymbol{\Xi}_{m} \boldsymbol{\Theta} & i \boldsymbol{\Xi}_{m} \boldsymbol{\Theta} & i \tilde{\boldsymbol{\Xi}}_{m} \boldsymbol{\Theta} & -\hat{\boldsymbol{\Xi}}_{m} \boldsymbol{\Theta} & -\check{\boldsymbol{\Xi}}_{m} \boldsymbol{\Theta}
\end{array}\right] \\
& \mathbf{P} \triangleq \operatorname{diag}\left\{\left[\begin{array}{lllll}
\mathbf{I} & \boldsymbol{\Lambda} & \boldsymbol{\Lambda} & \boldsymbol{\Lambda} & \boldsymbol{\Lambda}
\end{array}\right]\right\} \in \mathbb{R}^{5 K \times 5 K}, \\
& \boldsymbol{\Xi}_{m} \triangleq\left[\begin{array}{ccc}
\xi_{1}^{m, t_{0}} & \cdots & \xi_{K}^{m, t_{0}} \\
\vdots & \ddots & \vdots \\
\xi_{1}^{m, t_{N-1}} & \cdots & \xi_{K}^{m, t_{N-1}}
\end{array}\right] \text {, } \\
& \tilde{\boldsymbol{\Xi}}_{m} \triangleq \tilde{\mathbf{T}} \boldsymbol{\Xi}_{m}, \hat{\boldsymbol{\Xi}}_{m} \triangleq \hat{\mathbf{T}} \boldsymbol{\Xi}_{m}, \check{\boldsymbol{\Xi}}_{m} \triangleq \check{\mathbf{T}} \boldsymbol{\Xi}_{m}, \\
& \boldsymbol{\Theta} \triangleq \operatorname{diag}\left\{\left[e^{i b_{1}}, \cdots, e^{i b_{K}}\right]\right\}, \\
& \boldsymbol{\Lambda} \triangleq \operatorname{diag}\left\{\left[a_{1}, \cdots, a_{K}\right]\right\} \text {, } \\
& \tilde{\mathbf{T}} \triangleq \operatorname{diag}\left\{\left[t_{0}, \cdots, t_{N-1}\right]\right\} \text {, } \\
& \hat{\mathbf{T}} \triangleq \operatorname{diag}\left\{\left[\left|t_{0}-\tau\right|, \cdots,\left|t_{N-1}-\tau\right|\right]\right\}, \\
& \check{\mathbf{T}} \triangleq \operatorname{diag}\left\{\left[\left(t_{0}+2 \tau m\right), \cdots,\left(t_{N-1}+2 \tau m\right)\right]\right\} \text {. }
\end{aligned}
$$

Stacking the derivatives from each echo $m$, yields $\partial \mathbf{x} / \partial \boldsymbol{\gamma}=\mathbf{Q P}$, where

$$
\mathbf{Q}=\left[\begin{array}{lll}
\mathbf{Q}_{0}^{T} & \cdots & \mathbf{Q}_{M-1}^{T}
\end{array}\right]^{T} \in \mathbb{C}^{N M \times 5 K} .
$$

Table 1. Parameters for simulated data

\begin{tabular}{lcccc}
\hline$k$ & 1 & 2 & 3 & 4 \\
\hline$f_{k}(\mathrm{~Hz})$ & 0.0329 & 0.0122 & 0.0049 & -0.0232 \\
$\beta_{k}(\mathrm{~Hz})$ & 0.0202 & 0.0077 & 0.0053 & 0.0035 \\
$\eta_{k}\left(10^{-3}\right)$ & 0.1811 & 0.2647 & 0.2130 & 0.2221 \\
$\left|\alpha_{k}\right|$ & 1.20 & 5.00 & 4.30 & 3.65 \\
$\angle \alpha_{k}(\mathrm{rad})$ & 0.4591 & -2.8045 & 0.0661 & -1.9922 \\
\hline
\end{tabular}

The FIM thus becomes

$$
\operatorname{FIM}(\gamma)=\frac{2}{\sigma^{2}} \mathbf{P} \operatorname{Re}\left\{\mathbf{Q}^{H} \mathbf{Q}\right\} \mathbf{P}
$$

implying that

$$
\operatorname{CRB}(\gamma)=\frac{\sigma^{2}}{2} \mathbf{P}^{-1}\left[\operatorname{Re}\left\{\mathbf{Q}^{H} \mathbf{Q}\right\}\right]^{-1} \mathbf{P}^{-1} .
$$

Letting

$$
\boldsymbol{\Gamma} \triangleq\left[2 \operatorname{Re}\left\{\mathbf{Q}^{H} \mathbf{Q}\right\}\right]^{-1}
$$

yields the further simplified expression for the sought CRBs

$$
\begin{aligned}
\operatorname{CRB}\left(a_{k}\right) & =[\boldsymbol{\Gamma}]_{k k} a_{k}^{2} / \mathrm{SNR}_{k} \\
\operatorname{CRB}\left(b_{k}\right) & =[\boldsymbol{\Gamma}]_{(k+K)(k+K)} / \mathrm{SNR}_{k} \\
\operatorname{CRB}\left(\omega_{k}\right) & =[\boldsymbol{\Gamma}]_{(k+2 K)(k+2 K)} / \mathrm{SNR}_{k} \\
\operatorname{CRB}\left(\beta_{k}\right) & =[\boldsymbol{\Gamma}]_{(k+3 K)(k+3 K)} / \mathrm{SNR}_{k} \\
\operatorname{CRB}\left(\eta_{k}\right) & =[\boldsymbol{\Gamma}]_{(k+4 K)(k+4 K)} / \mathrm{SNR}_{k},
\end{aligned}
$$

where $\mathrm{SNR}_{k}=a_{k}^{2} / \sigma^{2}$.

\section{NUMERICAL EXAMPLES}

The proposed algorithm was evaluated using simulated NQR data, formed as to mimic the response signal from the explosive TNT when excited using a PSL sequence. Such signals can be well modeled as a sum of four damped sinusoidal signals, with the parameters as detailed in Table 1 (see [6] for further details on the signal and the relevant measurement setup). Based on the typical setup examined in [6], we use $N=256$ measurements, for $M=32$ echoes, with $\tau=164$ and $t_{0}=36$, where the last two parameters are normalized with the sampling frequency and are therefore unit-less. The algorithm was evaluated using the normalized root mean squared error (NRMSE), defined as:

$$
\mathrm{NRMSE}=\sqrt{\frac{1}{P} \sum_{p=1}^{P}\left(\frac{\hat{x}_{p}}{x}-1\right)^{2}},
$$

where $x$ denotes the true parameter value and $\hat{x}_{p}$ the estimate of this parameter. The signal-to-noise ratio (SNR) is defined as $\sigma_{s}^{2} \sigma^{-2}$, with $\sigma_{s}^{2}$ and $\sigma^{2}$ denoting the power of the noise-free signal and the noise variance, respectively. Moreover, we use $L=\tilde{N} / 2$, where $\tilde{N}$ denotes the number of samples of either the expanding or the decaying part of the echo. With the used $\tau$, one obtains a symmetric echo [6], so that $L^{(+)}=L^{(-)}=64$. Fig. 2 shows the results from $P=500$ Monte-Carlo simulations for the fourth spectral line (the performance for the other lines was similar). As is common for ESPRIT-based estimators, it can be noted that the ET-ESP estimate does not fully reach the CRB and is therefore not statistically efficient. However, the difference is very small down to $\mathrm{SNR}=0$ 


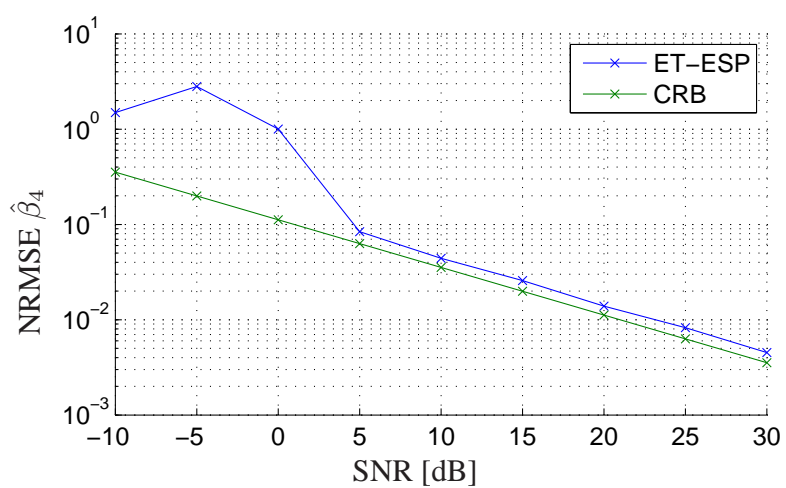

(a) NRMSE for estimates of $\beta_{4}$ and the CRB as given in (33).

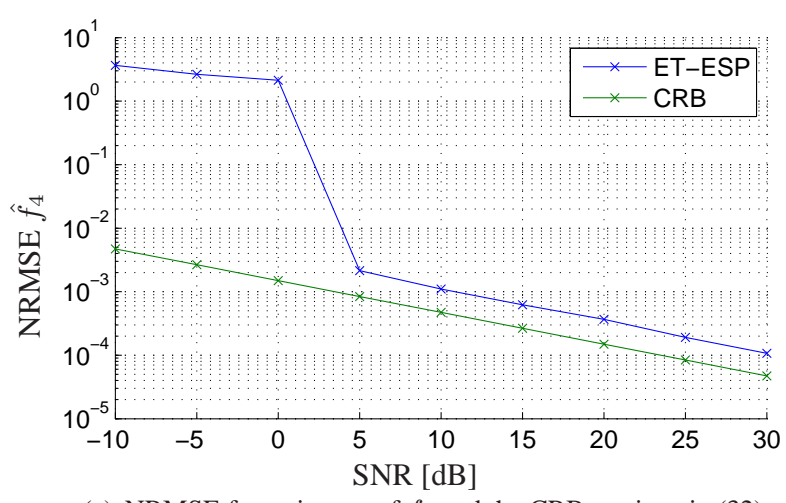

(c) NRMSE for estimates of $f_{4}$ and the CRB as given in (32).

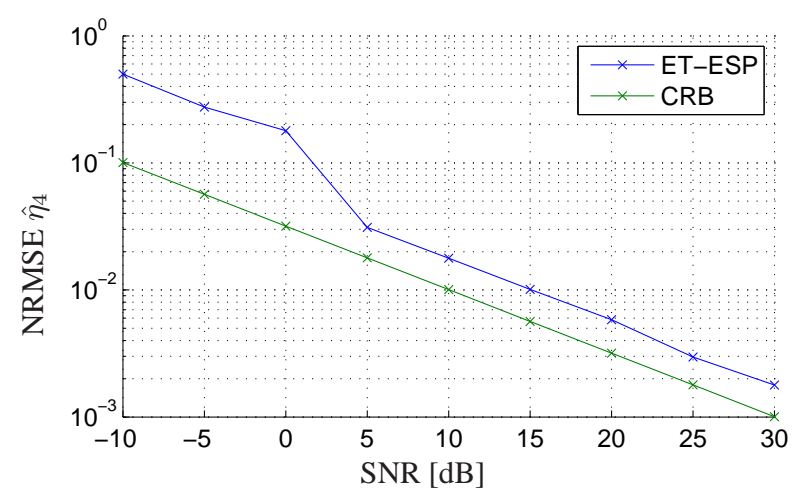

(b) NRMSE for estimates of $\eta_{4}$ and the CRB as given in (34).

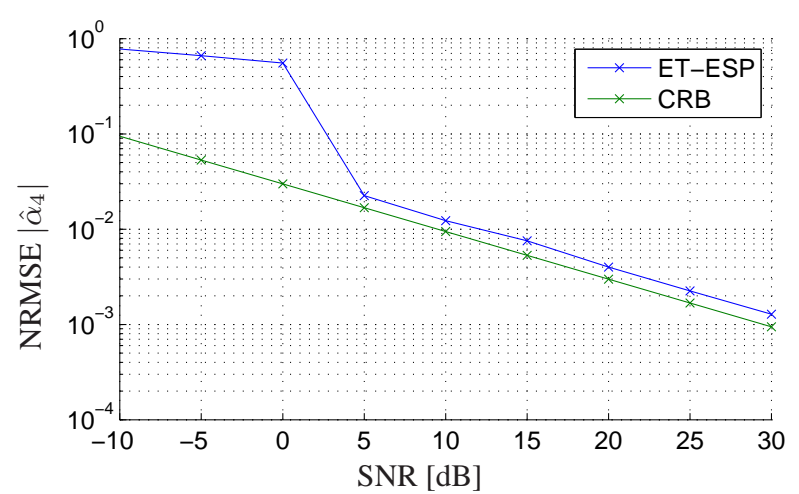

(d) NRMSE for estimates of $\left|\alpha_{4}\right|$ and the CRB as given in (30).

Fig. 2. NRMSE given by the proposed estimator for different parameters, compared with the respective CRB. Here, the NRMSE is empirically evaluated over 500 Monte-Carlo realizations, for symmetric echoes, with $N=256$ and $M=32$.

$\mathrm{dB}$ (which corresponds to $\sigma^{2} \approx 6$ ), before which the estimation error becomes very large. For $\mathrm{SNR}=5 \mathrm{~dB}$, the estimation error for the frequency $f_{4}$ is about $0.2 \%$, whereas for the damping coefficient, $\beta_{4}$, and the damping coefficient, $\eta_{4}$, it is about $8 \%$ and $3 \%$, respectively. The amplitude error $\left|\alpha_{4}\right|$ is about $2 \%$.

\section{CONCLUSIONS}

In this paper, we have derived an ESPRIT-based estimator and the corresponding CRB for the data model detailing the typically damped sinusoidal signals obtained in magnetic resonance measurements when formed using PSL data sequences. The estimator is computationally efficient and only requires the number of sinusoids to be known, which is typically the case in the considered applications. Via Monte-Carlo simulations, we have shown that the algorithm is close to being statistically efficient for typical signalto-noise ratios. The proposed method offers an attractive alternative as a standalone estimator or as an initial estimator for further refined estimates based on gradient or search-based techniques.

\section{REFERENCES}

[1] P. Stoica and R. Moses, Spectral Analysis of Signals, Prentice Hall, Upper Saddle River, N.J., 2005.
[2] Y. Hua and T. K. Sarkar, "Matrix pencil method for estimating parameters of exponentially damped/undamped sinusoids in noise," IEEE Trans. Acoust., Speech and Signal Process., vol. 38, no. 5, pp. 814-824, May 1990.

[3] A. Jakobsson, M. Mossberg, M. Rowe, and J. A. S. Smith, "Exploiting Temperature Dependency in the Detection of NQR Signals," IEEE Trans. Signal Process., vol. 54, no. 5, pp. 16101616, May 2006.

[4] A. Gregorovič and T. Apih, "TNT detection with 14N NQR: Multipulse sequences and matched filter," J. Magn. Reson., vol. 198, no. 2, pp. 215-221, June 2009.

[5] H. Chen, S. Van Huffel, A. van den Boom, and P. van den Bosch, "Extended HTLS methods for parameter estimation of multiple data sets modeled as sums of exponentials," in 13th Int. Conf. Digit. Signal Process., Santorini, 1997, vol. 2, pp. 1035-1038.

[6] S. D. Somasundaram, Advanced Signal Processing Algorithms Based on Novel Nuclear Quadrupole Resonance Models for the Detection of Explosives, Ph.D. thesis, King's College London, London, United Kingdom, 2007.

[7] Y. Yao and S. P. Pandit, "Cramér-Rao Lower Bound for a Damped Sinusoidal Process," IEEE Trans. Signal Process., vol. 43, no. 4, pp. 878-885, April 1995. 